\title{
Laser-Driven Direct Synthesis of Carbon Nanodots and Application as Sensitizers for Visible-light Photocatalysis
}

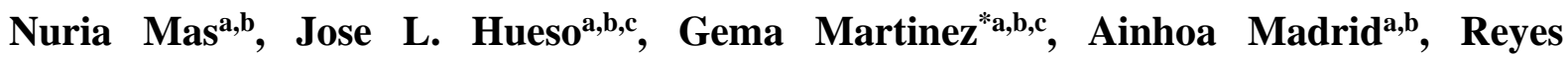 \\ Mallada $^{a, b, c}$, M. Carmen Ortega-Liebana ${ }^{a, b}$, Carlos Bueno-Alejo ${ }^{a, b}$, and Jesus \\ Santamaria ${ }^{* a, b, c}$ \\ ${ }^{a}$ Institute of Nanoscience of Aragon (INA) and Department of Chemical and Environmental \\ Engineering, Campus Rio Ebro, 50018, Zaragoza, Spain
}

${ }^{b}$ Networking Research Center on Bioengineering, Biomaterials and Nanomedicine (CIBERBBN), 28029, Madrid, Spain

${ }^{c}$ Instituto de Ciencia de Materiales de Aragon (ICMA), Consejo Superior de Investigaciones Científicas (CSIC-Universidad de Zaragoza), Zaragoza, Spain

*Corresponding authors. E-mail: gemamar@unizar.es (Gema Martinez); jesus.santamaria@unizar.es (Jesus Santamaria)

\begin{abstract}
We present the first successful synthesis of monodisperse carbon nanodots (CNDs) with tunable photoluminescence (PL) carried out by laser pyrolysis of two common volatile organic precursors such as toluene and pyridine. Remarkably, the initial chemical composition of the precursor determines the formation of undoped or N-doped CNDs and their corresponding absorption response in the visible range (expanded for the latter). We demonstrate the control and versatility of this synthesis method to tune the final outcome and its potential to explore a
\end{abstract}


great number of potential solvent candidates. Furthermore, we have successfully exploited these CNDs (both undoped and $\mathrm{N}$-doped) as effective sensitizers of $\mathrm{TiO}_{2}$ nanoparticles in the visible-light driven photo-degradation of a cationic dye selected as model organic pollutant.

\section{INTRODUCTION}

Luminescent carbon-based nanostructures such as graphene dots, carbon nanotubes (CNTs) or carbon nanodots (CNDs) have attracted much attention in recent years on account of their outstanding optical properties and potential applications [1-5]. Broad range optical response is strongly desirable in applications such as bioimaging, sensing, solar cells and theranostics [2, 4, 6-20]. Furthermore, their use as photosensitizers to expand the photocatalytic response of semiconductors beyond the UV range has also attracted a great attention in photocatalysis in the Advanced Oxidation Process (AOP) of organic pollutants present in wastewaters $[2,4,11,20-41]$. These features make CNDs an excellent alternative to semiconductor quantum dots (QDs) or up-converting rare-earth based nanoparticles (UCNPs) that are mostly based on scarce or potentially more toxic components. CNDs are typically synthesized by wet chemistry routes (i.e. microwave or hydrothermal methods), which provide high control of particle size distributions. [6, 22, 35, 42-44]. However, these methods normally demand extended reaction times and yield limited quantities. Furthermore, additional costly and tedious post-synthetic treatments are typically required due to the lack of chemical composition homogeneity [45-47]. Alternatively, CNDs have been also produced by laser driven methods involving either the laser ablation of solid target in organic solution [48-51], the laser fragmentation of power carbon material $[51,52]$ or alternatively the laser fragmentation of powdered carbon materials $[51,52]$. These routes constitute a single step strategy that does not require the use of external chemical agents, guarantying the high-purity of synthesized CNDs. However, these approaches, alike other top-down methods, generally 
face the major drawback of an intrinsically wide size distribution of the produced nanoparticles and low production efficiency [51, 53]. For instance, classical laser fragmentation only leads to the reduction of almost half of the original powder carbon material $[54,55]$. Additionally, these methods display limited availability of control in stoichiometry requirements, making them very restricted processes for the synthesis of doped CNDs. Among the laser synthesis methods, laser pyrolysis represents an elegant alternative for the one-pot synthesis of a wide variety of carbonaceous materials, including amorphous carbon materials, fullerenes, carbon black, carbides or even graphene powders In the process of $\mathrm{CO}_{2}$ laser pyrolysis $[45,56-60]$ the reaction proceeds in-between the laser beam and the molecular flow of gaseous/vapors phase reactants. The resultant condensable products are generated at the interface. The process is based on the energy coupling between the laser light and the precursor's mixture. In case of no absorbing gas precursors an additional substance the so-called sensitizer, has to be introduced among the reactants [61]. Compared with other laser-driven approaches, the laser pyrolysis allows: (i) a well-defined interaction volume delimited by the radiation cross-section and the emerging gas flow; (ii) spatial uniformity of the reaction zone; (iii) highly localized and faster heating (leading to rapid nucleation) with faster quenching of the particles grow and (iv) versatile adjusting of process parameters to endow the nanoparticle with different composition and tailored properties. Although the use of a laser source represents an attractive alternative to conventional synthesis methods of CNDs (i. e. continuous production, potential scalability, low purification needs) $[58-60,62]$, to date it has not been possible to retrieve them as freestanding and well-dispersed nanoparticles.

Herein we present, to the best of our knowledge, the first example of highly monodisperse undoped and $\mathrm{N}$-doped CNDs with tuneable optical response synthesized by laser pyrolysis. Furthermore, we have tested these CNDs as sensitizers to extend the photocatalytic response of titania beyond the UV range [21, 25-31, 35, 41] in the photodegradation of an organic dye 
[63-65]. Volatile organic solvents (toluene and pyridine) were selected as carbon precursors, whereas the generated nanoparticles after laser irradiation were collected in a liquid media to prevent coalescence events $[45,56,57]$. With this selection we aimed at evaluating the potential $\mathrm{N}$-doping effects caused by the subtle modification of the chemical composition of the organic precursors (by introducing an $\mathrm{N}$ heteroatom). In this regard, the addition of co-reactants containing $\mathrm{N}, \mathrm{P}$ or $\mathrm{S}$ atoms has been used to promote the sensitizing capabilities of CNDs in conventional (e.g. hydrothermal) synthesis [8] but not achieved before through laser-driven pyrolysis. In addition, we evaluate the predominant role of the generated CNDs as electron donors with capacity to expand the absorption of anatase towards the visible range.

\section{EXPERIMENTAL}

\subsection{Chemicals}

Toluene (99.7\%), pyridine (99.8\%), triethylene glycol (TREG, 99\%), titanium(IV) oxide 99.8\% anatase (< $200 \mathrm{~nm}$ pseudo spherical nanopowders), crystal violet dye and ethanol with analytical purity grade were purchased from Sigma-Aldrich and used without any further purification.

\subsection{Laser-driven synthesis of carbon nanodots (undoped CNDs and N-doped CNDs)}

The synthesis of the CNDs was carried out by laser pyrolysis technology. The continuous gas flow reactor and the liquid collection system have been described elsewhere $[45,56,57]$. The first step was the optimization of the process parameters. In general, the most relevant process parameters governing the synthesis include working pressure, gas flow rates, concentration of the precursor in the reactant stream and laser intensity $[45,56,57]$. The overall operating pressure and the gas flow rates determine the time exposure in the laser beam. Both variables tend to shift the residence time in opposite direction. For instance, increasing the total pressure 
reduces the total volumetric flow rate and consequently increases the residence time (provided that the rest of parameters are fixed). In addition to these two parameters, the precursor feeding temperature (in case of liquid precursors) determines the final concentration of precursor in the reactant stream. All these key variables control the formation of gas phase aggregates and the final nanoparticle sizes. On the other hand, the laser power and the gas sensitizer (i.e. $\mathrm{SF}_{6}$ ) feeding flow rate directly correlate with the temperatures reached in the reaction zone. Increasing both parameters favor an overall increase of the reaction temperature. In order to avoid further contaminations derived from the partial decomposition of the sensitizer, it is important to keep as low as possible the gas sensitizer gas flow rates. The optimized reaction conditions used in this work are listed in Table S1 (see Supporting Information). Toluene (in the case of the undoped CNDs) or pyridine (for N-doped CNDs) were selected as carbon precursors. Both liquid solvents fulfill the requirements and can be easily fed into the reaction chamber due to their sufficiently high vapor pressure [66]. Moreover, the thermal process in which these precursor molecules are converted into atoms, releases $\mathrm{C} \mathrm{sp}^{2}$ (toluene) and $\mathrm{C} \mathrm{sp} / \mathrm{N}$ (pyridine), making them suitable candidates for the synthesis of carbon nanodots and further evaluation of the potential $\mathrm{N}$-doping effects. The high temperature required for the decomposition of those precursors $[67,68]$ led to use $250 \mathrm{~W}$ of laser power in order to reach the corresponding dissociation threshold, promoting at the same time the formation of highly crystalline undoped CNDs and N-doped CNDs. Lower laser power values typically yielded either amorphous or pseudo-crystalline carbonaceous networks. The volatile organic solvents were fed from a sealed glass vessel equipped with regulatory opening-close valves and immersed in a temperature-controlled bath. A flow rate of $30 \mathrm{sccm}$ of sulfur hexafluoride $\left(\mathrm{SF}_{6}\right)$ selected as sensitizer that specifically absorbs the laser infrared wavelength, and $130 \mathrm{sccm}$ of argon (Ar) was used as carrier gas passing through the organic precursor reservoir. An infrared $\mathrm{CO}_{2}$ laser beam (Rofin $\mathrm{SCx} 30, \lambda=10.6 \mu \mathrm{m}$ ) was settled to intersect orthogonally with the gas- 
phase organic reactant streams. The feeding inlet consisted of two concentric nozzles; the reactive gas flow (toluene/ $\mathrm{SF}_{6}$ or pyridine $\left./ \mathrm{SF}_{6}\right)$ entered through the central inner tube $(6.35 \mathrm{~mm}$ external diameter and $3.28 \mathrm{~mm}$ internal diameter), while an argon flow (coaxial gas) was fed through the external concentric tube (12.7 $\mathrm{mm}$ external diameter and $9.5 \mathrm{~mm}$ internal diameter). The coaxial argon stream (flow rate: $100 \mathrm{sccm}$ ) has the role of confining the reactant (precursor/SF6 mixture) and the nucleated particles in a region close to the flow axis, minimizing deposition on the chamber walls. Additional Ar (600 sccm) and $\mathrm{N}_{2}(200 \mathrm{sccm})$ streams were used to provide a protective sheath on the vertical and horizontal windows of the chamber. They also helped to avoid nanoparticle deposition onto the walls. All gas flow rates to the reactor were mass-flow controlled (Hytec). The working pressure in the reactor chamber was controlled through a diaphragm valve, located between the reaction chamber and the vacuum pump. The as-prepared nanoparticles were directly collected in a TREG container. Total reaction times were defined for 6 hours. During the laser pyrolysis process, the TREG solution progressively darkened, as a clear signal of successful capture of the generated product materials. The resulting suspensions were labeled as CNDs and N-CNDs depending on the precursor used for their synthesis, toluene or pyridine, respectively. In order to carry out further characterization of the solids, the obtained suspension was rinsed with ethanol by centrifugation at $15000 \mathrm{rpm}$ and $15^{\circ} \mathrm{C}$ for $30 \mathrm{~min}$ to remove the excess of TREG solvent. The resulting suspensions were stored at room temperature until further use. The synthesis of these nanomaterials has been performed by the Synthesis of Nanoparticles Unit of the ICTS "NANBIOSIS" at the Institute of Nanoscience of Aragon (INA)-Universidad de Zaragoza.

\subsection{Carbon nanodots decorating $\mathrm{TiO}_{2}$ nanoparticles}

$\mathrm{N}-\mathrm{CNDs} @ \mathrm{TiO}_{2}$ nanohybrids were obtained via a drying-vacuum step at $90{ }^{\circ} \mathrm{C} .0 .2 \mathrm{~g}$ of $\mathrm{TiO}_{2}$ anatase (which was previously heated treated at $180{ }^{\circ} \mathrm{C}$ during 2 hours) were suspended in $2 \mathrm{~mL}$ of ethanol. Then $1 \mathrm{~mL}$ of N-CNDs suspended in ethanol was added. This suspension 
was stirred vigorously for 1 hour at room temperature. After that the suspension was washed with water and centrifuged at $8000 \mathrm{rpm}$. The final solid was storaged after drying in a vacuumdriven oven at $90{ }^{\circ} \mathrm{C}$ during 1 hour.

\subsection{Characterization techniques}

The morphologies and particle size distributions were determined by transmission electron microscopy (TEM) (FEI Tecnai T20, operating at $200 \mathrm{kV}$ ). The high-resolution TEM images were obtained in a Titan (FEI TITAN ${ }^{3}$, operated at $300 \mathrm{kV}$ and equipped with a Gatan Image Filter (GIF Tridiem 863)). To prepare the samples, the nanoparticle suspensions were diluted into deionized water and then dropcasted onto a holey carbon TEM grid. The surface chemical composition of CNDs was analyzed by X-ray photoelectron spectroscopy (XPS) with an Axis Ultra DLD (Kratos Tech.) A monochromatic Al Ka source (1486.6 eV) was employed with multiple runs at $12 \mathrm{kV}, 10 \mathrm{~mA}$ and pass energy of $20 \mathrm{eV}$ was used. The binding energies were calibrated to the internal C1s $(284.3 \mathrm{eV})$ standard. Analyses of the peaks were performed with CasaXPS software, using a weighted sum of Lorentzian and Gaussian component curves after

Shirley background subtraction as described previously in the literature [69-74]. Steady-state fluorescence emission spectra and photoluminescence excitation (PLE) survey spectra were collected on a JASCO FP-6500 spectrofluorometer equipped with a $450 \mathrm{~W}$ xenon lamp for excitation, with temperature controller ETC-273T at $25{ }^{\circ} \mathrm{C}$, using $5 \times 10 \mathrm{~mm}$ cuvettes and a LS55 Fluorescence Spectrometer (PerkinElmer) equipped with a xenon arc lamp as the light source and a quartz cell (10 x $10 \mathrm{~mm})$. UV-Vis spectroscopy (V-67, Jasco Company) was used to analyze the absorbance spectra of the nanomaterials and the decoloration of crystal violet with a quartz cell of $1 \mathrm{~cm}$ light path. A 90 Plus Particle size analyzer (Brookhaven Instruments Corporation) was employed to determine $\zeta$-potential measurements. The fluorescence quantum

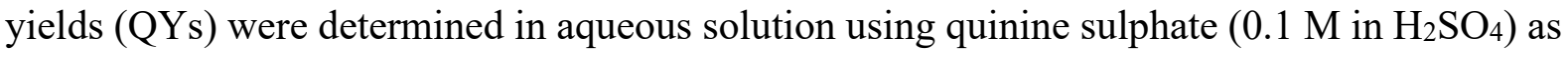
reference fluorophore $[6,75]$. Using excitation wavelengths at $360 \mathrm{~nm}$ and keeping absorbance 
values below 0.1 , QYs were 5\% and 12\% for undoped and N-doped CNDs, respectively and in line with previously reported carbon dots [20, 35, 76-78]. The surface morphology and Zheights of the CNDs was characterized by atomic force microscopy (AFM) using a MultiMode 8 AFM system (Bruker). Images were taken in tapping mode using a high-resolution force modulation "Golden" silicon cantilevers (FMG01 series) with integrated tetrahedral tip, exhibiting a typical force constant and a resonant frequency of $3 \mathrm{~N} \mathrm{~m}^{-1}$ and $60 \mathrm{kHz}$, respectively. Raman characterization was carried out using a Raman Spectrometer WiTec alpha 300. A Helium-Neon $532 \mathrm{~nm}$ laser was used for excitation of the Raman signal and the laser power for each sample was $1 \mathrm{~mW}$. A 50x optical aperture was used resulting in a $1.2 \mu \mathrm{m}$ diameter spot. Acquisition times of 1 second and a single spectrum accumulation per spectrum were typically required. Raman fitting of the raw spectra was carried out using 4 bands denoted as G, D, D'and I following the convention established in previous literature [79-81] to establish the specific contributions of ordered and disordered fractions of the carbon network.

\subsection{Photocatalytic degradation of Crystal Violet under white-LED irradiation}

Photo-catalytic degradation assays of the cationic organic dye (see Figure S6 for $\zeta$-potential measurements) crystal violet were performed under white light LED irradiation. A white light LED emitter from LED-Engin (9.37 W, LZ4 model) was coupled to Synjet@ a cooling system. The degradation assays were carried out suspending $0.6 \mathrm{mg}$ of the $\mathrm{TiO}_{2}$ decorated with the different CNDs or plain $\mathrm{TiO}_{2}$ nanoparticles as control, in $3 \mathrm{~mL}$ of $0.025 \mathrm{mM}$ Crystal Violet $(\mathrm{CV})$ solution at its natural operating $\mathrm{pH}(\mathrm{pH}=6)[63-65,82]$. The selection of this cationic dye was suitable due to the negative surface charge measured for the N-CNDs@ $\mathrm{TiO}_{2}$ nanoparticles by $\zeta$-potential at that specific $\mathrm{pH}$ value. This charge differences would in principle favor a stronger electrostatic interaction between the photocatalyst and the organic molecule. The suspensions were magnetically stirred under dark for $30 \mathrm{~min}$, prior to the irradiation with a white light emitting LED, in order to guarantee the establishment of an 
adsorption/desorption equilibrium. Different aliquots of $200 \mu \mathrm{L}$ were taken at different time intervals. After centrifugation, the samples were measured by UV-visible spectroscopy monitoring the maximum absorbance of $\mathrm{CV}$ centred at $588 \mathrm{~nm}$. All the photodegradation assays of $\mathrm{CV}$ were performed under the same experimental conditions. At least three assays were carried out for each tested solid and different N-CNDs@ $\mathrm{TiO}_{2}$ batches were tested in order to certify the reproducibility of the photocatalytic effect depending on the synthesized material.

\subsection{Detection of radicals and quenching experiments under LED irradiation}

The generation of hydroxyl radical under white LED irradiation was evaluated using terephtalic acid (TA) as a probe, which selectively reacts with $\bullet \mathrm{OH}$ to form a fluorescent derivative $[35$, $78,83]$. In a typical procedure, the detection of hydroxyl radicals $(\bullet \mathrm{OH})$ was carried out with the aid of terephthalic acid (TA, $3 \mathrm{~mL}, 5 \mathrm{mM}$ ), which selectively reacts to generate a fluorescent product (2-hydroxy terephthalic acid) emitting at ca. $425 \mathrm{~nm}$. After white LED illumination at different time intervals, the mixture solution was centrifuged to remove the photocatalyst nanoparticles. The fluorescence emission spectrum of the generated 2-hydroxy disodium terephtalate in the supernatant was subsequently measured at an excitation wavelength of 315 $\mathrm{nm}$. The quenching experiments of the photocatalytic activity were carried out by degradation of $\mathrm{CV}$ under white LED light irradiation. $0.6 \mathrm{mg}$ nanohybrids were suspended in $3 \mathrm{~mL}$ that contained $0.025 \mathrm{mM} \mathrm{CV}$ and $1 \mathrm{mM}$ of EDTA-Na2 (selected as hole scavenger) or alternatively $1 \mathrm{mM}$ 1-butanol (as hydroxyl radical scavenger) in an aqueous suspension at $\mathrm{pH}$. The suspensions were magnetically stirred under dark for $30 \mathrm{~min}$, prior to the irradiation with one white light emitting LED, in order to guarantee the establishment of an adsorption/desorption equilibrium. Different aliquots of $200 \mu \mathrm{L}$ were taken at several selected time intervals and after centrifugation of the samples these were measured in UV-visible spectroscopy equipment, following the decreasing of the maximum absorption peak of the dye centred at $588 \mathrm{~nm}$. 


\section{RESULTS AND DISCUSSION}

\subsection{Synthesis and characterization of undoped and N-doped carbon dots retrieved by laser}

pyrolysis of different organic volatile solvents

Scheme1 summarizes the laser pyrolysis process used to generate the CNDs $[45,56,57]$. The resulting condensable products are generated from the laser induced chemical reaction at the interface of the laser beam and vaporized solvent (i. e. toluene for undoped or pyridine for $\mathrm{N}$-doped CNDs). Sulfur hexafluoride is used as photosensitiser to accomplish the energy transfer process between the laser light and precursor's mixture. The net result of the laser excitation is a thermal process in which the reactant molecules are converted into atoms (Scheme 1 bottom, Reaction Zone). CNDs formation starts abruptly when a sufficient concentration of condensable products is reached in the vapor phase. To prepare small and spherical particles it is necessary to create a high degree of super-saturation for inducing the formation of a high density of nuclei and then quickly quench the particle growth by slowing down the kinetics, due to the fast decrease of temperature with radial distance. After leaving the hot reaction zone gas, the as-prepared nanoparticles are driven by the gas flow into the collector system containing TREG (Scheme 1 bottom, Collection System). 

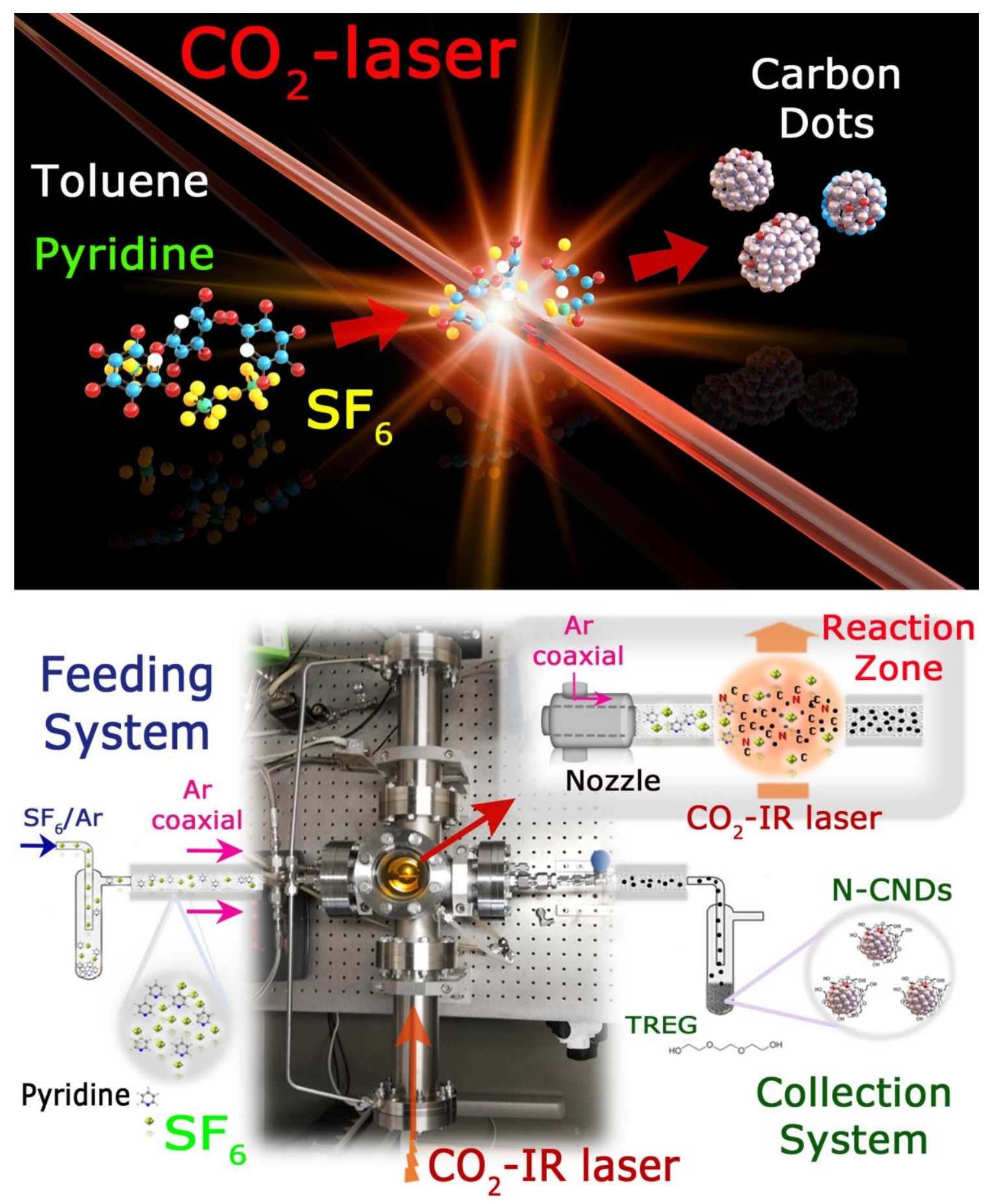

Scheme 1. (Top): Schematic display of the $\mathrm{CO}_{2}$ laser assisted pyrolysis process to generate carbon nanodots derived from toluene or pyridine; (Bottom): Detailed scheme of the experimental setup including the feeding system, the reaction area and the collection in TREG medium. 
Transmission electron microscopy (TEM) analysis of the collected suspensions showed a narrow size distribution of nanoparticles with mean diameters of $2.3 \pm 1.6 \mathrm{~nm}$ and $2.0 \pm 0.7$ $\mathrm{nm}$ for the CNDs derived from toluene (Figure S1 and Figure S2a) and pyridine (Figure 1a and Figure S2b), respectively. HR-TEM revealed that both CNDs are highly crystalline (Figure 1a$1 \mathrm{~b}$ and Figure S1a-S1b) stacked in a hexagonal crystal structure corresponding to graphite $2 \mathrm{H}$ with a space group P6_3mc $[84,85]$. These structures were confirmed on multiple individual CNDs by FFT images that rendered lattice distances of $0.184 \mathrm{~nm}, 0.199 \mathrm{~nm}$ and $0.210 \mathrm{~nm}$ corresponding to (102), (101) and (100) planes of graphite 2H (see Figure 1c and Figure S1), respectively $[84,85]$. Given the two-dimensional nature of TEM, the shape of the CNDs was further assessed by Atomic Force Microscopy (AFM). Figure 1d showed the 3D and 2D AFM topographic images of the $\mathrm{N}$-doped CNDs. The location of $\mathrm{A}, \mathrm{B}$, and $\mathrm{C}$ were randomly spotted dots, their heights were 1.5, 2.1 and $2.9 \mathrm{~nm}$, respectively, yielding and average height of 2.2 $\mathrm{nm}$, which is close to the size $(2.0 \mathrm{~nm})$ obtained from TEM. An analogous dimension organization was observed in the case of the undoped CNDs (Figure S3) and confirmed the pseudo-spherical distribution of the CNDs.

Representative first-order Raman spectra revealed two maxima for both CNDs centered at $\sim 1350 \mathrm{~cm}^{-1}$ and $\sim 1595 \mathrm{~cm}^{-1}$, respectively (see Figure S4). The additional broadening observed for the N-doped CNDs was mostly caused by an asymmetric tailing at lower shift values. The spectra were deconvoluted following previous fitting parameters by Maldonado et al. [80] or Cuesta et al. [79]. The fitting bands were referred to as G, D', D and I, respectively. The G band at $1595 \mathrm{~cm}^{-1}$ arising from vibrational mode of ordered graphitic crystal planes with $\mathrm{sp}^{2}$ bonds was only slightly more intense for the N-CNDs than the undoped ones (Figure S4) and accounted for a similar level of ordered graphitic domains. In contrast, the contributions of D and especially $\mathrm{D}^{\prime}$ and I bands that are attributed to relaxations in local symmetry of graphitic planes caused by local distortions, stacking faults, the presence of impurities or the presence of 
disordered graphitic planes is more prominent in the Raman spectra recorded for the N-doped CNDs (Figure S4) [79-81, 86]. An analogous evolution has been previously observed in Ndoped CNTs with increasing $\mathrm{N}$ levels [80].

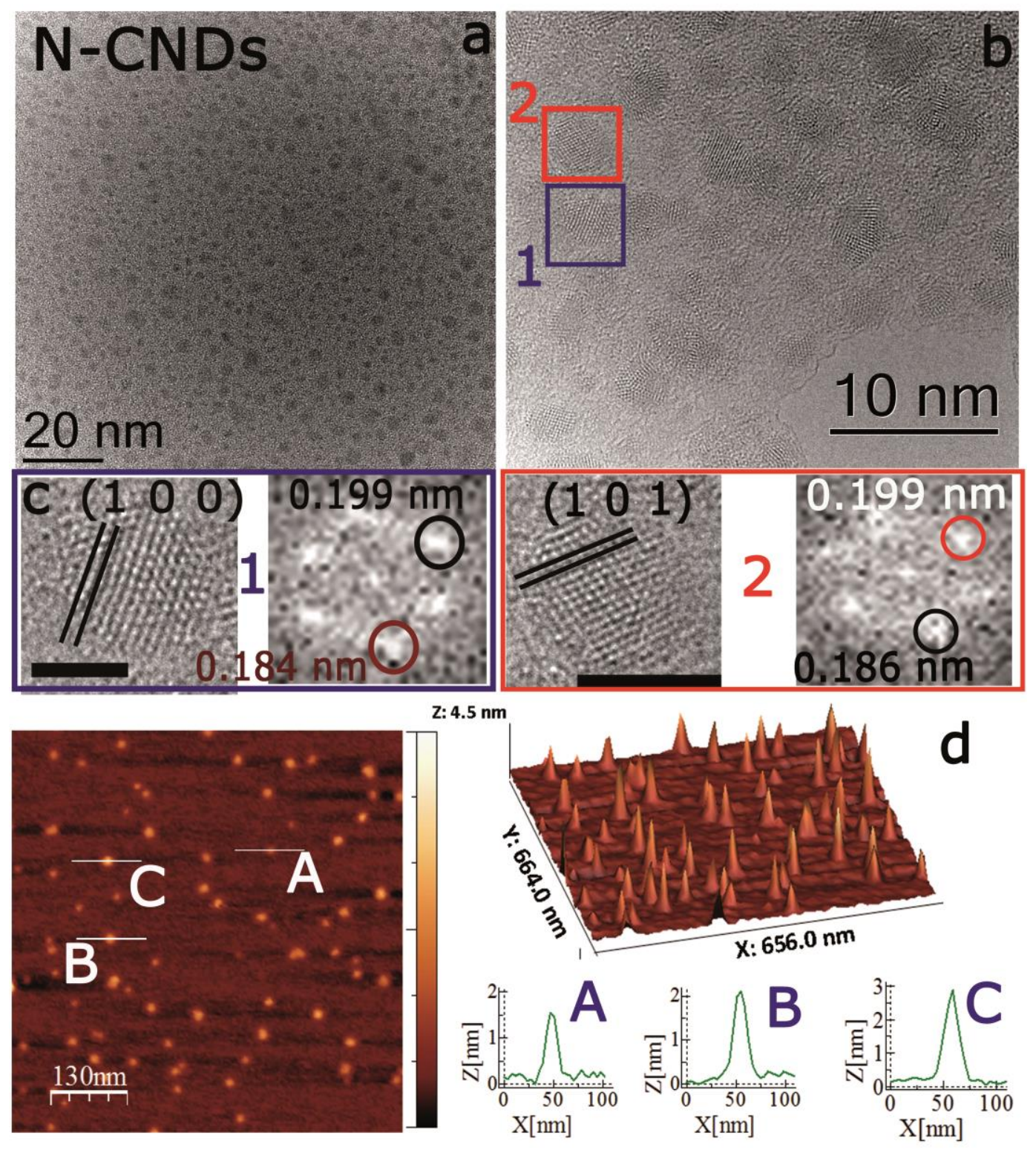

Figure 1. Morphological characterization of the N-doped carbon nanodots (N-CNDs) retrieved from the laser-assisted pyrolysis of pyridine: a) Low-magnification TEM image accounting for the homogeneous distribution; b) HR-TEM image and inset accounting for the crystalline nature of the N-CNDs; c) 2D and 3D AFM topographic images including Z-height analysis at positions marked with blue lines and labelled as $\mathrm{A}-\mathrm{C}$ in the $2 \mathrm{D}$ image of the NCNDs. 
Moreover, the N-doping was confirmed by XPS analysis (Figure 2, Figures S5-S6). The presence of $\mathrm{N} 1 \mathrm{~s}$ signal further confirmed the formation of $\mathrm{N}-\mathrm{C}$ bonds in the carbon nanoparticles retrieved from pyridine (Figure 2b). In contrast, the CNDs from toluene rendered no signal in this region (Figure S5). N1s photoemission spectrum in the N-CNDs was fitted with two characteristics peaks (Figure 2b). The first contribution at $399.4 \mathrm{eV}$ was attributed to pyridinic-N (N-6), while the second centered at $401.7 \mathrm{eV}$ was assigned to graphitic-N quaternary amines (N-Q) (Figure 2b) $[13,16,17,26,70,72-75,83,87]$. The surface atomic percentage detected for $\mathrm{N}$ was slightly above $1 \%$ (Table $\mathrm{S} 2$ ), thereby accounting for a limited doping level for the N-CNDs. In the expanded C1s spectrum of N-CNDs (Figure 2a) the dominant peak at $284.3 \mathrm{eV}$ raised from C-C bond with $\mathrm{sp}^{2}$ hybridization, while the peaks at around 285.7, and $288.5 \mathrm{eV}$ are attributed to $\mathrm{C}-\mathrm{N} / \mathrm{C}-\mathrm{O}$, and $\mathrm{C}=\mathrm{O}$ and/or $\mathrm{C}=\mathrm{N}$ bonds, respectively $[69,70,75,87,88]$. As expected, when $\mathrm{N}$ atoms are intercalated within the graphitic lattices the signals in the spectrum of $\mathrm{C} 1 \mathrm{~s}$ changed accordingly. Therefore, a less pronounced peak at $286.2 \mathrm{eV}(\mathrm{C}-\mathrm{N} / \mathrm{C}-\mathrm{O})$ was observed for the CNDs derived from toluene (Figure S5). Analogous expanded O1s XP spectra were found for both type of carbon dots (Figure 2c and Figure S5). The O1s peaks at 531.3 and $532.9 \mathrm{eV}$ shown in Figure 2c were associated with oxygen doubly and singly bound to aromatic rings, respectively $[13,69,71$, $72,74]$. The major presence of surface oxygen in the N-doped CNDs (Table S2) points out to a major presence of carbon with defects containing surface active groups in accordance with the major disorder level detected by Raman spectroscopy (Figure S4). The contribution of oxygen can be tentatively attributed with the oxygen present in the solvent precursors. Finally, it is worth mentioning the minor presence of $\mathrm{S}$ and $\mathrm{F}$ induced by the partial decomposition of the sensitizer (Table S2). 

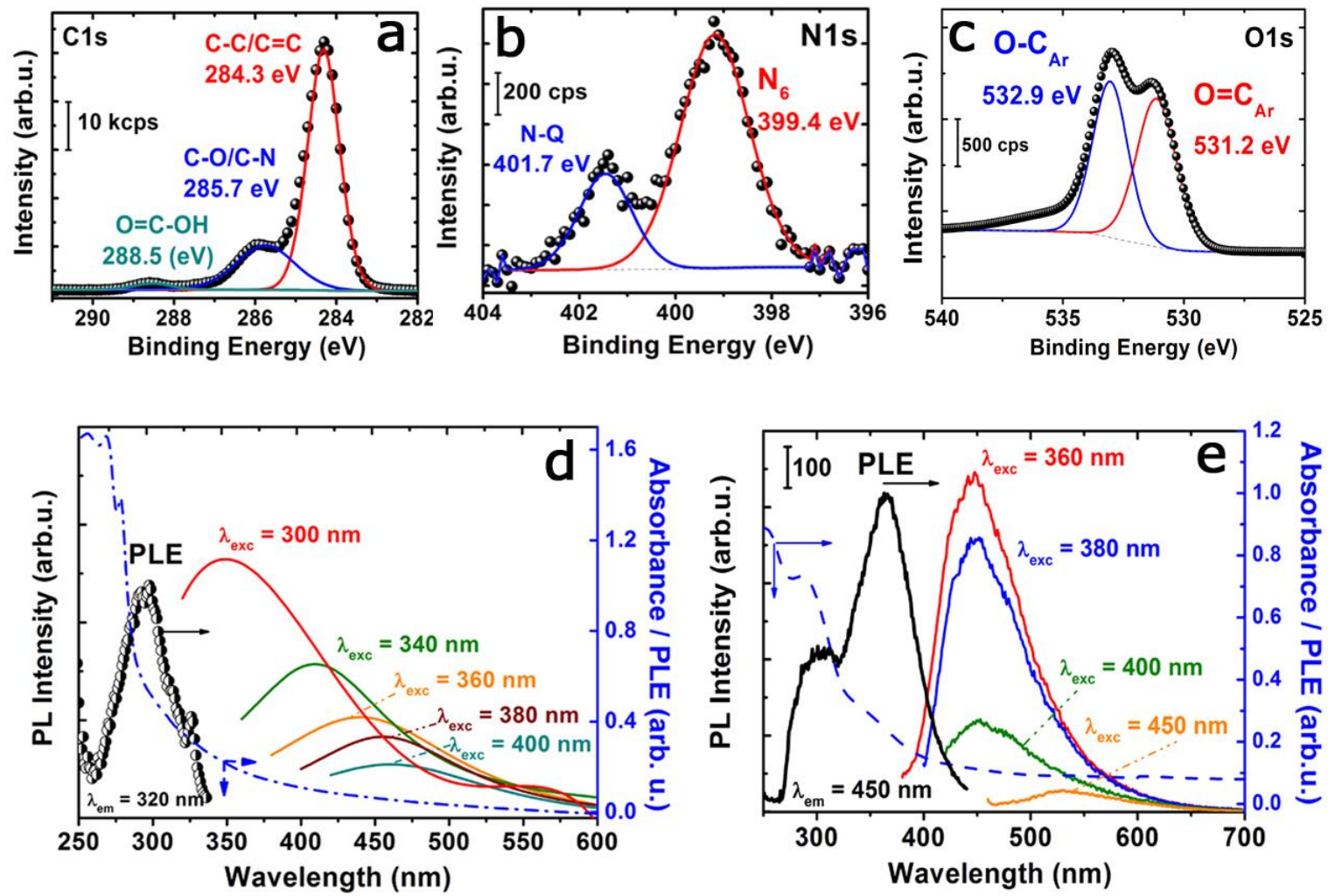

Figure 2. X-ray photoemission spectra and fittings of the a) $\mathrm{C} 1 \mathrm{~s}$ region; b) N1s region; c)

O1s region corresponding to the N-CNDs; d) Optical characterization of the undoped CNDs from toluene: Absorption spectrum, (blue dashed line), photoluminescence excitation spectrum at $\lambda_{\mathrm{em}}=320 \mathrm{~nm}$ (PLE-black dotted curve) combined with the photoluminescence (PL) spectra for a range of excitation wavelengths (300-400 nm); e) Optical characterization of the N-doped CNDs from pyridine: Absorption spectrum (blue dashed line), photoluminescence excitation spectrum at $\lambda_{\mathrm{em}}=450 \mathrm{~nm}$ (PLE-black curve) combined with the photoluminescence (PL) spectra for a range of excitation wavelengths $(360-450 \mathrm{~nm})$.

The absorption spectrum of the CNDs showed the typical $\pi-\pi^{*}$ transition band at $250-275$ $\mathrm{nm}$ [26] while in the N-doped CNDs a shoulder at $350 \mathrm{~nm}$ is typically assigned to the $\mathrm{n}-\pi^{*}$ transitions of the $\mathrm{N}$ centers (Figures 2d-2e) [1, 25, 26]. The broad absorption in the visible range has been previously attributed to a wider distribution of $\mathrm{N}$ levels and/or the presence of oxygen functional groups present in surface defects $[5,16,20,26,31,47,76,85,89,90]$. 
Therefore, in our case the doping with $\mathrm{N}$ atoms seems to disrupt the graphitic ordering in the CNDs and contributes to the creation of major fraction of disordered $\mathrm{sp}^{2}-\mathrm{sp}^{3}$ clusters within the main graphitic network. This hypothesis has been further supported by XPS and Raman analysis (vide supra) and in previous works in the recent literature [16, 28, 31, 91-94]. The evaluation of the optical properties corroborated the excitation wavelength dependence on the photoluminescence (PL) emission properties for the undoped CNDs (Figure 2d) that is tentatively attributed to optical selection of differently sized nanoparticles (quantum effect), surface traps and/or reorganization of solvent's polarization [95]. In the case of N-CNDs such dependence is not observed (Figure 2e), suggesting a different electronic configuration in which the radiative decay of excited electrons occur from $\mathrm{N}$ energy states of the N-CNDs [2426], due to the relatively strong electron affinity of $\mathrm{N}$ atoms. Indeed, previous experimental observations and quantum-mechanical calculations have proved the strong electronwithdrawing ability of the $\mathrm{N}$ atoms within the conjugated $\mathrm{C}$ plane $[24-26,96,97]$. This is confirmed again with the photoluminescence excitation spectra (PLE) (Figure 2e) in which the major contribution come from the wavelength corresponding to the $n-\pi *$ transitions at $350 \mathrm{~nm}$ in contrast with the corresponding excitation spectrum of the undoped CNDs where the major contribution comes from the $\pi-\pi^{*}$ (Figure $2 \mathrm{~d}$ ). This illustrates, to the best of our knowledge, i) the first examples of freestanding CNDs synthesized through the laser-assisted pyrolysis of regular solvents and how a minimal variation on the chemical structure of these starting precursors can be crucial to tune the optical response of the resulting products.

As an example of the functionality afforded by the CNDs, they have been applied in expanded-range photocatalysis. Taking into account that carbon nanostructures have been previously reported as successful sensitizers to expand the response of $\mathrm{TiO}_{2}$ beyond the $\mathrm{UV}$ region [27-30, 98-100], we used impregnation followed by drying under vacuum to deposit the undoped and N-doped CNDs onto commercial anatase nanoparticles (Figure 3a) [21]. HRTEM 
analysis confirmed the homogeneous distribution of the N-CNDs onto the semiconductor phase (Figure 3b). Likewise, an enhanced absorption towards the visible-NIR range was also observed by UV-Vis spectroscopy following the deposition of N-CNDs on anatase nanoparticles (Figure 3c). This is attributable to the contribution of extra $n-\pi^{*}$ transitions provided by the additional $\mathrm{N}$ energy surface states that will contribute to narrow the band gap of the hybrid material $[25,26]$.
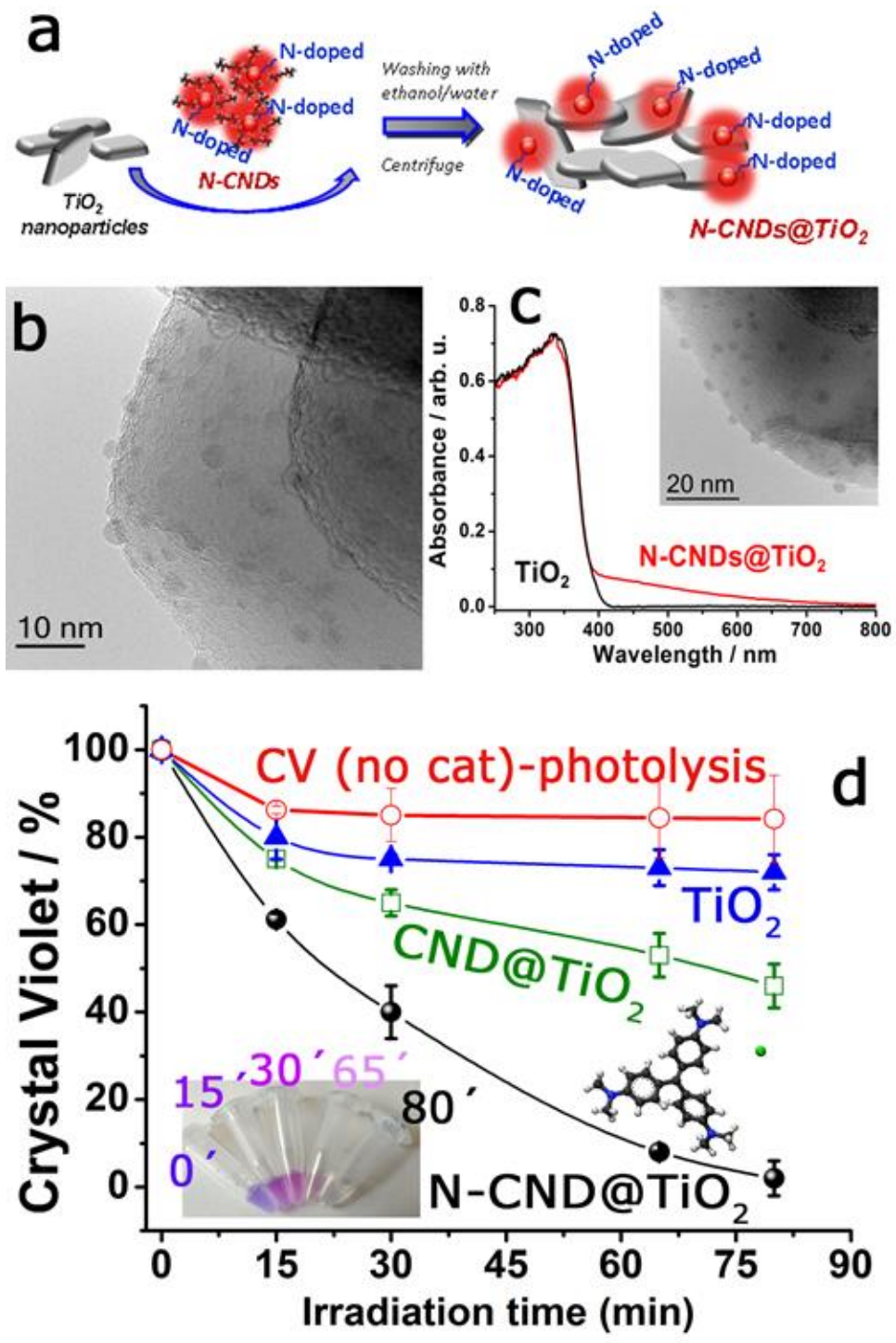

Figure 3. a) Scheme representing the incorporation of the $\mathrm{N}-\mathrm{CNDs}$ onto $\mathrm{TiO}_{2}$ nanoparticles surface to obtain the final N-CNDs@ $\mathrm{TiO}_{2}$ hybrid nanomaterial; b) HR-TEM image of the carbon nanodots retrieved from pyridine (N-CNDs) after deployment onto the $\mathrm{TiO}_{2}$ surface $(\mathrm{N}-$ CNDs@ $\mathrm{TiO}_{2}$ ); c) UV-visible absorbance spectra of $\mathrm{TiO}_{2}$ powder nanoparticles with and 
without N-CNDs; d) Crystal violet photodegradation curves under white LED light irradiation, in the absence of catalyst (void circles), with $\mathrm{TiO}_{2}$ nanoparticles (triangles) and in the presence of the undoped CNDs@ $\mathrm{TiO}_{2}$ (squared symbols) and N-CNDs@ $\mathrm{TiO}_{2}$ hybrids (filled circles), respectively. Inset: Crystal violet structure and digital pictures of different CV aliquots after irradiation at different time intervals in the presence of the N-CNDs@ $\mathrm{TiO}_{2} \mathrm{NPs}$.

\subsection{Evaluation of the Carbon Dots as Visible-light Photo-sensitizers}

The visible-light driven photo-activity of plain anatase nanoparticles, $\mathrm{CND}-\mathrm{TiO}_{2}$ and $\mathrm{N}$ CND- $\mathrm{TiO}_{2}$ hybrids was evaluated in the degradation of an organic model dye such as crystal violet $(\mathrm{CV})$ subjected to illumination with a high intensity white-light emitting LED (see Experimental section for details). As previously stated in the Experimental section, the photodegradation experiments were carried out at $\mathrm{pH}=6$, as typically reported for $\mathrm{CV}$ [63-65, 82]. The $\zeta$-Potential for the N-CNDs@ $\mathrm{TiO}_{2}$ nanoparticles at pH 6 (negatively charged, see Figure S6) would be beneficial to ensure an optimal electrostatic interaction with the cationic dye. Figure $3 \mathrm{~d}$ shows the complete degradation of $\mathrm{CV}$ after $80 \mathrm{~min}$ of irradiation in the presence of the N-CNDs@ $\mathrm{TiO}_{2}$ photocatalyst. In contrast, the control experiment with no catalyst or the non-decorated $\mathrm{TiO}_{2}$ support only rendered $10 \%$ and $40 \%$ maximum degradation, respectively under identical irradiation conditions. The hybrid containing the undoped CNDs achieved a maximum $\mathrm{CV}$ conversion of $50 \%$ after $80 \mathrm{~min}$, thereby confirming the enhanced photodegradation capabilities of the N-CNDs (Figure 3d).

Two additional consecutive photo-degradation experiments were carried out with the most active photocatalyst. After replacing with fresh dye solutions, the stability and reusability of the N-CNDs@ $\mathrm{TiO}_{2}$ were tested. There were no evidences of deactivation over three cycles (Figure S7). In order to establish a mechanism, we studied the steady-state fluorescence changes of disodium terephthalate (NaTA) in the presence of the $\mathrm{TiO}_{2}$ and N-CNDs@ $\mathrm{TiO}_{2}$ under white LED irradiation, respectively $[35,78]$. NaTA is a highly sensitive and selective 
fluorescent probe for hydroxyl radicals $(\cdot \mathrm{OH})$ (inset in Figure $4 \mathrm{a})$. The weakly fluorescent NaTA can react with $\cdot \mathrm{OH}$ and convert into the highly fluorescent 2-hydroxy disodium terephtalate [33, 35, 83]. Preliminary control experiments evaluating the direct photolysis of NaTA under white LED irradiation or the potential catalytic effect of $\mathrm{N}-\mathrm{CNDs} @ \mathrm{TiO}_{2}$ in the the absence of light were also carried out. No signal accounting for the generation of hydroxyl radicals was found (data not shown). Figure 4 shows how the PL signal of hydroxylated NaTa byproduct is quite more prominent in the photocatalyst decorated with N-CNDs than in the plain $\mathrm{TiO}_{2}$ NPs (Figure 4a). This corroborates the enhanced radical generation under visible light exposure when N-CNDs are present as sensitizers. Additional experiments with the in situ generation of hydroxyl radicals under white LED light irradiation have been also monitored using terephtalic acid (TA) as a probe, thereby corroborating the selective formation of these -OH radical groups only in the presence of the N-CNDs@ $\mathrm{TiO}_{2}$. 

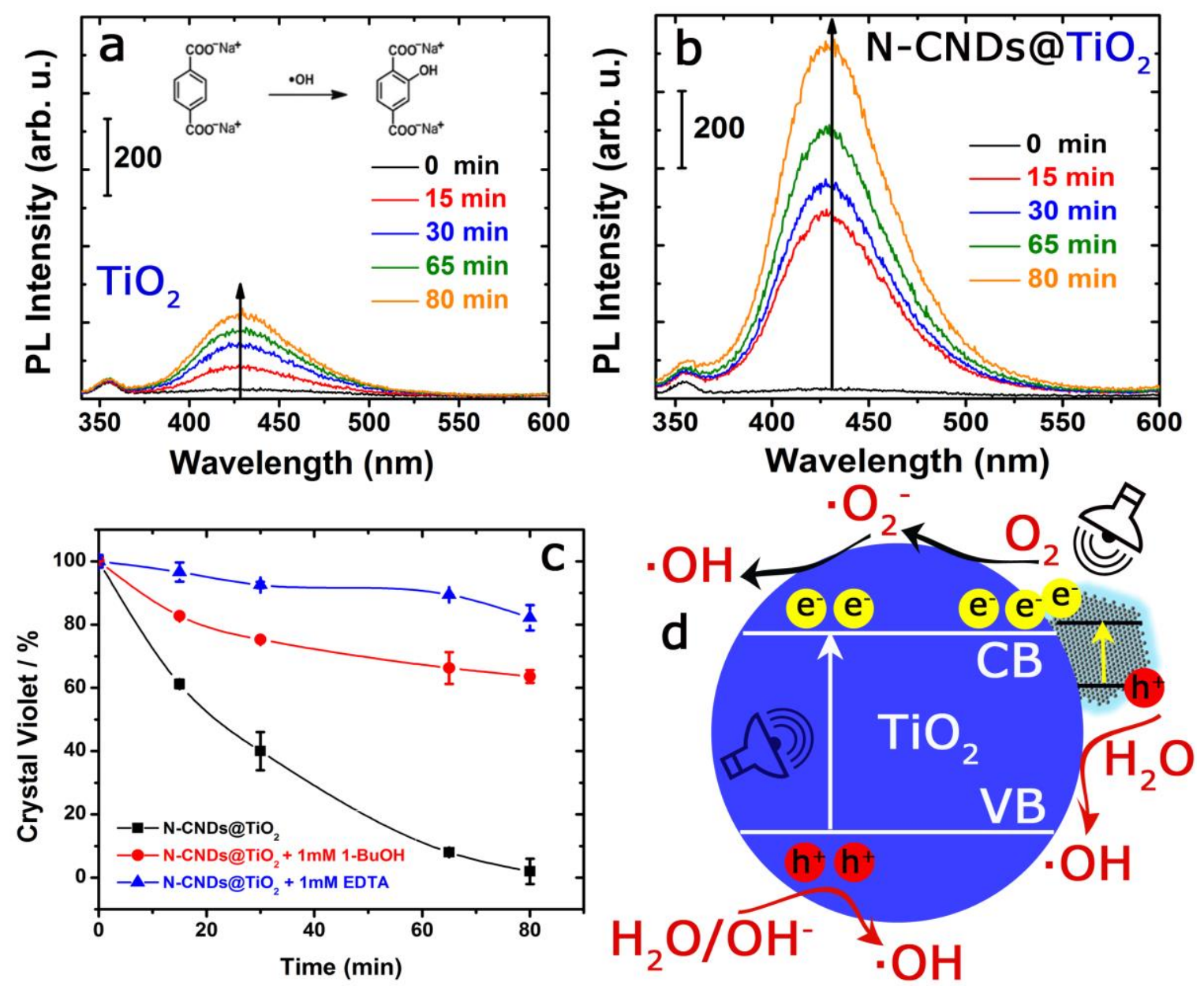

Figure 4. Evaluation of the in situ generation of hydroxyl radicals upon photoirradiation with a high radiance white LED in the presence of a) plain anatase commercial nanoparticles and b) N-CNDs@ $\mathrm{TiO}_{2}$ nanohybrids; c) Quenching experiments to evaluate the influence of hole (EDTA) and hydroxyl radical (butanol) scavengers; d) Schematic display of the role of CNDs as photosensitizers able to withdraw electrons to the conduction band of the titania support and the main reaction pathways to form hydroxyl radicals.

Additional experiments including different and specific scavengers (butanol for hydroxyl radicals and EDTA for holes) have been carried out in order to corroborate the active participation of these reactive oxidative species [101]. Given the much stronger quenching effect of EDTA (Figure 4c), the mechanism seems to be preferentially driven by the 
photogeneration of holes. In our opinion, the presence of holes can be favored due to the strong interaction between the CNDs and the titania support that overlap their energy levels and facilitate a proper electron transfer from the excited levels of CNDs to the conduction band of $\mathrm{TiO}_{2}$ (Figure 4d) $[24,27,102]$. As a result, the electron-hole recombination rate in the CNDs is delayed and the holes can readily react with water molecules to form hydroxyl radicals $\left(\mathrm{h}^{+}\right.$ $+\mathrm{H}_{2} \mathrm{O} \rightarrow \cdot \mathrm{OH}+\mathrm{H}^{+}$, Figure $\left.4 \mathrm{~d}\right)$ ), either on the surface of the CNDs or through the surface of Ti-O-Ti species as claimed by Nosaka et al. [103]. The other ROS formation mechanism governed by the interaction between photo-generated electrons and adsorbed oxygen molecules to yield oxygen superanions (e- $+\mathrm{O}_{2}$ (ads) $\rightarrow \cdot \mathrm{O}_{2}{ }^{-} \rightarrow \ldots \rightarrow \cdot \mathrm{OH}$, see Figure $4 \mathrm{~d}$ ) is less straightforward and requires a major number of intermediate reactions to form the most reactive hydroxyl radicals $[63,65]$. It is also worth mentioning a small fraction of deep UV light from LED is also able to excite the $\mathrm{TiO}_{2}$ itself (Figure $3 \mathrm{~d}$ and Figure 4a). Still, the positive effect of both undoped and N-doped CNDs is clearly demonstrated (Figure 3d).

\section{CONCLUSIONS}

In summary, for the first time we have shown that luminescent CNDs with tunable optical properties can be obtained through the laser-driven transformation of conventional organic solvents such as toluene and pyridine. Upon laser irradiation, the pyrolysis of toluene primarily led to the conformation of carbon nanodots with oxygen functional groups while the nitrogen-containing precursor gave rise to an $\mathrm{N}$-doped carbon species with expanded optical range towards the visible range. The process is extremely fast and provides an optimal result, as the doping elements are intimately embedded in the carbon structures. This novel approach (laser-driven decomposition of organic molecules with the desired doping elements) harbours enormous prospects compared with classical preparation techniques for light-emitting CNDs, such as hydrothermal synthesis. In this case, the functionality of the carbon dots as efficient 
sensitizers to expand the photocatalytic response of anatase paves the way for multiple alternatives of synthesis where controlled doping with heteroatoms is relevant.

\section{Acknowledgements}

Financial support from the European Research Council (ERC Advanced Grant number 742684) is gratefully acknowledged. We also acknowledge the support from NANOLIGHT (REA grant number 294094), CIBER-BBN and MINECO (Spain) with project CTQ201679419-R. The synthesis of materials has been performed by the Platform of Production of Biomaterials and Nanoparticles of the NANOBIOSIS ICTS, more specifically by the Nanoparticle Synthesis Unit of the CIBER in Bioengineering, Biomaterials \& Nanomedicine (CIBER-BBN). The TEM studies were conducted at the Laboratorio de Microscopias Avanzadas, Instituto de Nanociencia de Aragon, Universidad de Zaragoza, Spain. We thank Dr. Silvia Irusta for her help in the XPS measurements.

\section{Electronic Supplementary Material}

Supplementary material (PDF) is available in the online version of this article. TEM images of the carbon dots retrieved from toluene (Figure S1); Particle size histograms determined from TEM images (Figure S2); AFM topographic images of the carbon nanodots (CNDs) synthesized by laser pyrolysis of toluene (Figure S3); Raman Spectra of the CNDs (Figure S4); XPS survey spectra (Figure S5); Fitted X-ray photoelectron spectra of undoped CNDs (Figure S6); $\zeta$-potential of $\mathrm{TiO}_{2}$ and $\mathrm{N}-\mathrm{CNDs} @ \mathrm{TiO}_{2}$ at different $\mathrm{pH}$ intervals (Figure S7); Evaluation of the reusability of the N-CNDs@ $\mathrm{TiO}_{2}$ photocatalyst (Figure S8).

\section{Competing financial interests}

The authors declare no competing financial interests. 


\section{REFERENCES}

[1] S.N. Baker, G.A. Baker, Luminescent Carbon Nanodots: Emergent Nanolights, Angew. Chem.-Int. Edit. 49 (2010) 6726-6744.

[2] S.Y. Lim, W. Shen, Z.Q. Gao, Carbon quantum dots and their applications, Chem. Soc. Rev. 44 (2015) 362-381.

[3] C.M. Zhang, J. Lin, Defect-related luminescent materials: synthesis, emission properties and applications, Chem. Soc. Rev. 41 (2012) 7938-7961.

[4] Q.J. Xiang, J.G. Yu, M. Jaroniec, Graphene-based semiconductor photocatalysts, Chem. Soc. Rev. 41 (2012) 782-796.

[5] S.T. Yang, Y.S. Ho, Research performance and trends of fluorescent carbon nanoparticles: a science citation index expanded-based analysis, J Nanopart Res 21 (2019) 112.

[6] M.C. Ortega-Liebana, M.M. Encabo-Berzosa, M.J. Ruedas-Rama, J.L. Hueso, Nitrogen-Induced Transformation of Vitamin C into Multifunctional Up-converting Carbon Nanodots in the Visible-NIR Range, Chem.-Eur. J. 23 (2017) 3067-3073.

[7] J.Y. Qin, J.P. Huo, P.Y. Zhang, J. Zeng, T.T. Wang, H.P. Zeng, Improving the photocatalytic hydrogen production of $\mathrm{Ag} / \mathrm{g}-\mathrm{C} 3 \mathrm{~N} 4$ nanocomposites by dye-sensitization under visible light irradiation, Nanoscale 8 (2016) 2249-2259.

[8] D. Qu, M. Zheng, P. Du, Y. Zhou, L.G. Zhang, D. Li, H.Q. Tan, Z. Zhao, Z.G. Xie, Z.C. Sun, Highly luminescent S, N co-doped graphene quantum dots with broad visible absorption bands for visible light photocatalysts, Nanoscale 5 (2013) 12272-12277.

[9] S.J. Zhu, Q.N. Meng, L. Wang, J.H. Zhang, Y.B. Song, H. Jin, K. Zhang, H.C. Sun, H.Y. Wang, B. Yang, Highly Photoluminescent Carbon Dots for Multicolor Patterning, Sensors, and Bioimaging, Angew. Chem.-Int. Edit. 52 (2013) 3953-3957. 
[10] M.J. Ruedas-Rama, A. Orte, E.A.H. Hall, J.M. Alvarez-Pez, E.M. Talavera, Quantum dot photoluminescence lifetime-based pH nanosensor, Chem. Commun. 47 (2011) 2898-2900.

[11] X.T. Zheng, A. Ananthanarayanan, K.Q. Luo, P. Chen, Glowing Graphene Quantum Dots and Carbon Dots: Properties, Syntheses, and Biological Applications, Small 11 (2015) $1620-1636$.

[12] A.B. Bourlinos, A. Stassinopoulos, D. Anglos, R. Zboril, M. Karakassides, E.P. Giannelis, Surface functionalized carbogenic quantum dots, Small 4 (2008) 455-458.

[13] M.C. Ortega-Liebana, N.X. Chung, R. Limpens, L. Gomez, J.L. Hueso, J. Santamaria, T. Gregorkiewicz, Uniform luminescent carbon nanodots prepared by rapid pyrolysis of organic precursors confined within nanoporous templating structures, Carbon 117 (2017) 437 446.

[14] P. Roy, A.P. Periasamy, C.Y. Lin, G.M. Her, W.J. Chiu, C.L. Li, C.L. Shu, C.C. Huang, C.T. Liang, H.T. Chang, Photoluminescent graphene quantum dots for in vivo imaging of apoptotic cells, Nanoscale 7 (2015) 2504-2510.

[15] L.H. Shi, Y.Y. Li, X.F. Li, X.P. Wen, G.M. Zhang, J. Yang, C. Dong, S.M. Shuang, Facile and eco-friendly synthesis of green fluorescent carbon nanodots for applications in bioimaging, patterning and staining, Nanoscale 7 (2015) 7394-7401.

[16] K. Hola, M. Sudolska, S. Kalytchuk, D. Nachtigallova, A.L. Rogach, M. Otyepka, R. Zboril, Graphitic Nitrogen Triggers Red Fluorescence in Carbon Dots, ACS Nano 11 (2017) 12402-12410.

[17] K. Hola, Y. Zhang, Y. Wang, E.P. Giannelis, R. Zboril, A.L. Rogach, Carbon dotsEmerging light emitters for bioimaging, cancer therapy and optoelectronics, Nano Today 9 (2014) 590-603. 
[18] C.J. Reckmeier, Y. Wang, R. Zboril, A.L. Rogach, Influence of Doping and Temperature on Solvatochromic Shifts in Optical Spectra of Carbon Dots, J. Phys. Chem. C 120 (2016) 10591-10604.

[19] Y. Xiong, J. Schneider, C.J. Reckmeier, H. Huang, P. Kasak, A.L. Rogach, Carbonization conditions influence the emission characteristics and the stability against photobleaching of nitrogen doped carbon dots, Nanoscale 9 (2017) 11730-11738.

[20] G.A.M. Hutton, B.C.M. Martindale, E. Reisner, Carbon dots as photosensitisers for solar-driven catalysis, Chem. Soc. Rev. 46 (2017) 6111-6123.

[21] M.C. Ortega-Liebana, J.L. Hueso, S. Ferdousi, R. Arenal, S. Irusta, K.L. Yeung, J. Santamaria, Extraordinary sensitizing effect of co-doped carbon nanodots derived from mate herb: Application to enhanced photocatalytic degradation of chlorinated wastewater compounds under visible light, Appl. Catal. B-Environ. 218 (2017) 68-79.

[22] D.H. Wang, L. Jia, X.L. Wu, L.Q. Lu, A.W. Xu, One-step hydrothermal synthesis of $\mathrm{N}$-doped $\mathrm{TiO} 2 / \mathrm{C}$ nanocomposites with high visible light photocatalytic activity, Nanoscale 4 (2012) 576-584.

[23] M. Shamsipur, A. Barati, S. Karami, Long-wavelength, multicolor, and white-light emitting carbon-based dots: Achievements made, challenges remaining, and applications, Carbon 124 (2017) 429-472.

[24] Z.J. Zhang, T.T. Zheng, X.M. Li, J.Y. Xu, H.B. Zeng, Progress of Carbon Quantum Dots in Photocatalysis Applications, Part. Part. Syst. Charact. 33 (2016).

[25] R. Shi, Z. Li, H.J. Yu, L. Shang, C. Zhou, G.I.N. Waterhouse, L.Z. Wu, T.R. Zhang, Effect of Nitrogen Doping Level on the Performance of N-Doped Carbon Quantum Dot/TiO2 Composites for Photocatalytic Hydrogen Evolution, ChemSusChem 10 (2017) 4650-4656. 
[26] Y.Q. Dong, H.C. Pang, H.B. Yang, C.X. Guo, J.W. Shao, Y.W. Chi, C.M. Li, T. Yu, Carbon-Based Dots Co-doped with Nitrogen and Sulfur for High Quantum Yield and Excitation-Independent Emission, Angew. Chem.-Int. Edit. 52 (2013) 7800-7804.

[27] V. Binas, D. Venieri, D. Kotzias, G. Kiriakidis, Modified TiO2 based photocatalysts for improved air and health quality, J. Materiomics 3 (2017) 3-16.

[28] Y. Park, J. Yoo, B. Lim, W. Kwon, S.W. Rhee, Improving the functionality of carbon nanodots: doping and surface functionalization, J. Mater. Chem. A 4 (2016) 11582-11603.

[29] N.R. Nalid, A. Majid, M.B. Tahir, N.A. Niaz, S. Khalid, Carbonaceous-TiO2 nanomaterials for photocatalytic degradation of pollutants: A review, Ceram. Int. 43 (2017) $14552-14571$.

[30] R. Leary, A. Westwood, Carbonaceous nanomaterials for the enhancement of TiO2 photocatalysis, Carbon 49 (2011) 741-772.

[31] M. Han, S.J. Zhu, S.Y. Lu, Y.B. Song, T.L. Feng, S.Y. Tao, J.J. Liu, B. Yang, Recent progress on the photocatalysis of carbon dots: Classification, mechanism and applications, Nano Today 19 (2018) 201-218.

[32] V. Likodimos, Photonic crystal-assisted visible light activated $\mathrm{TiO} 2$ photocatalysis, Appl. Catal. B-Environ. 230 (2018) 269-303.

[33] M.C. Ortega-Liebana, J.L. Hueso, A. Larrea, V. Sebastiana, J. Santamaria, Feroxyhyte nanoflakes coupled to up-converting carbon nanodots: a highly active, magnetically recoverable, Fenton-like photocatalyst in the visible-NIR range, Chem. Commun. 51 (2015) $16625-16628$.

[34] X.W. Wang, G.Z. Sun, P. Routh, D.H. Kim, W. Huang, P. Chen, Heteroatom-doped graphene materials: syntheses, properties and applications, Chem. Soc. Rev. 43 (2014) 70677098. 
[35] M.C. Ortega-Liebana, J.L. Hueso, S. Ferdousi, K.L. Yeung, J. Santamaria, Nitrogendoped luminescent carbon nanodots for optimal photo-generation of hydroxyl radicals and visible-light expanded photo-catalysis, Diam. Relat. Mat. 65 (2016) 176-182.

[36] A.P. Bhirud, S.D. Sathaye, R.P. Waichal, J.D. Ambekar, C.J. Park, B.B. Kale, In-situ preparation of $\mathrm{N}-\mathrm{TiO} 2 /$ graphene nanocomposite and its enhanced photocatalytic hydrogen production by H2S splitting under solar light, Nanoscale 7 (2015) 5023-5034.

[37] H.T. Li, R.H. Liu, S.Y. Lian, Y. Liu, H. Huang, Z.H. Kang, Near-infrared light controlled photocatalytic activity of carbon quantum dots for highly selective oxidation reaction, Nanoscale 5 (2013) 3289-3297.

[38] M.B. Vasic, M.S. Randjelovic, M.Z. Momilovic, B.Z. Matovic, A.R. Zarubica, Degradation of crystal violet over heterogeneous $\mathrm{TiO} 2$-based catalysts: The effect of process parameters, Process. Appl. Ceram. 10 (2016) 189-198.

[39] Q.H. Yao, L.P. Lin, T.T. Zhao, X. Chen, Advances in Preparation, Physicochemical Properties and Applications of Heteroatom-Doped Graphene Quantum Dots, Prog. Chem. 27 (2015) 1523-1530.

[40] A. Hosseinnia, M. Keyanpour-Rad, M. Pazouki, Photo-catalytic degradation of organic dyes with different chromophores by synthesized nanosize TiO2 particles, World Appl Sci J. 8 (2010) 1327-1332.

[41] C.C. Chen, W.H. Ma, J.C. Zhao, Semiconductor-mediated photodegradation of pollutants under visible-light irradiation, Chem. Soc. Rev. 39 (2010) 4206-4219.

[42] H.P. Liu, T. Ye, C.D. Mao, Fluorescent carbon nanoparticles derived from candle soot, Angew. Chem.-Int. Edit. 46 (2007) 6473-6475.

[43] S.N. Qu, X.Y. Wang, Q.P. Lu, X.Y. Liu, L.J. Wang, A Biocompatible Fluorescent Ink Based on Water-Soluble Luminescent Carbon Nanodots, Angew. Chem.-Int. Edit. 51 (2012) 12215-12218. 
[44] X.F. Jia, J. Li, E.K. Wang, One-pot green synthesis of optically pH-sensitive carbon dots with upconversion luminescence, Nanoscale 4 (2012) 5572-5575.

[45] G. Martinez, A. Malumbres, R. Mallada, J.L. Hueso, S. Irusta, O. Bomati-Miguel, J. Santamaria, Use of a polyol liquid collection medium to obtain ultrasmall magnetic nanoparticles by laser pyrolysis, Nanotechnology 23 (2012) (9 pp) 425605.

[46] B.S. Chen, F.M. Li, S.X. Li, W. Weng, H.X. Guo, T. Guo, X.Y. Zhang, Y.B. Chen, T.T. Huang, X.L. Hong, S.Y. You, Y.M. Lin, K.H. Zeng, S. Chen, Large scale synthesis of photoluminescent carbon nanodots and their application for bioimaging, Nanoscale 5 (2013) 1967-1971.

[47] W. Ding, Z.D. Wei, S.G. Chen, X.Q. Qi, T. Yang, J.S. Hu, D. Wang, L.J. Wan, S.F. Alvi, L. Li, Space-Confinement-Induced Synthesis of Pyridinic- and Pyrrolic-Nitrogen-Doped Graphene for the Catalysis of Oxygen Reduction, Angew. Chem.-Int. Edit. 52 (2013) 1175511759.

[48] J. Lin, Z.W. Peng, Y.Y. Liu, F. Ruiz-Zepeda, R.Q. Ye, E.L.G. Samuel, M.J. Yacaman, B.I. Yakobson, J.M. Tour, Laser-induced porous graphene films from commercial polymers, Nat. Commun. 5 (2014) (8pp) 5714.

[49] T.N. Lin, K.H. Chih, C.T. Yuan, J.L. Shen, C.A.J. Lin, W.R. Liu, Laser-ablation production of graphene oxide nanostructures: from ribbons to quantum dots, Nanoscale 7 (2015) 2708-2715.

[50] R.Q. Ye, Y. Chyan, J.B. Zhang, Y.L. Li, X. Han, C. Kittrell, J.M. Tour, Laser-Induced Graphene Formation on Wood, Adv. Mater. 29 (2017) (7pp) 1702211.

[51] D.S. Zhang, B. Goekce, S. Barcikowski, Laser Synthesis and Processing of Colloids: Fundamentals and Applications, Chem. Rev. 117 (2017) 3990-4103. 
Alonso, G. Minguez-Vega, Fabrication by Laser Irradiation in a Continuous Flow Jet of Carbon Quantum Dots for Fluorescence Imaging, ACS Omega 3 (2018) 2735-2742.

[53] V. Amendola, M. Meneghetti, What controls the composition and the structure of nanomaterials generated by laser ablation in liquid solution?, Phys. Chem. Chem. Phys. 15 (2013) 3027-3046.

[54] S.L. Hu, K.Y. Niu, J. Sun, J. Yang, N.Q. Zhao, X.W. Du, One-step synthesis of fluorescent carbon nanoparticles by laser irradiation, J. Mater. Chem. 19 (2009) 484-488.

[55] X.Y. Li, H.Q. Wang, Y. Shimizu, A. Pyatenko, K. Kawaguchi, N. Koshizaki, Preparation of carbon quantum dots with tunable photoluminescence by rapid laser passivation in ordinary organic solvents, Chem. Commun. 47 (2011) 932-934.

[56] A. Malumbres, G. Martinez, R. Mallada, J.L. Hueso, O. Bomati-Miguel, J. Santamaria, Continuous production of iron-based nanocrystals by laser pyrolysis. Effect of operating variables on size, composition and magnetic response, Nanotechnology 24 (2013) (13 pp) 325603.

[57] A. Malumbres, G. Martinez, J.L. Hueso, J. Gracia, R. Mallada, A. Ibarra, J. Santamaria, Facile production of stable silicon nanoparticles: laser chemistry coupled to in situ stabilization via room temperature hydrosilylation, Nanoscale 7 (2015) 8566-8573.

[58] G. Martinez, A. Malumbres, A. Lopez, R. Mallada, J.L. Hueso, J. Santamaria, LaserAssisted Production of Carbon-Encapsulated Pt-Co Alloy Nanoparticles for Preferential Oxidation of Carbon Monoxide, Front. Chem. 6-Art.487 (2018) 10 pp.

[59] M.T. Swihart, Vapor-phase synthesis of nanoparticles, Curr. Opin. Colloid Interface Sci. 8 (2003) 127-133. 
[60] A.A. Puretzky, D.B. Geohegan, X. Fan, S.J. Pennycook, Dynamics of single-wall carbon nanotube synthesis by laser vaporization, Appl. Phys. A-Mater. Sci. Process. 70 (2000) 153-160.

[61] E. Borsella, S. Botti, L. Caneve, L. De Dominicisand, R. Fantoni, IR multiple-photon excitation of polyatomic molecules: a route towards nanostructures, Phys. Scr. 78 (2008).

[62] V. Sebastian, M. Arruebo, J. Santamaria, Reaction Engineering Strategies for the Production of Inorganic Nanomaterials, Small 10 (2014) 835-853.

[63] Y. Liu, X.Z. Liu, D.Z. Lu, P.F. Fang, R. Xiong, J.H. Wei, C.X. Pan, Carbon deposited TiO2-based nanosheets with enhanced adsorption ability and visible light photocatalytic activity, J. Mol. Catal. A-Chem. 392 (2014) 208-215.

[64] S. Senthilkumaar, K. Porkodi, Heterogeneous photocatalytic decomposition of Crystal Violet in UV-illuminated sol-gel derived nanocrystalline $\mathrm{TiO} 2$ suspensions, J. Colloid Interface Sci. 288 (2005) 184-189.

[65] C.C. Chen, F.D. Mai, K.T. Chen, C.W. Wu, C.S. Lu, Photocatalyzed N-de-methylation and degradation of crystal violet in titania dispersions under UV irradiation, Dyes Pigment. 75 (2007) 434-442.

[66] D.R. Lide (Ed.), CRC Handbook of Chemistry and Physics, 84th ed., CRC Press LLC: Boca Raton, 2003.

[67] N.R. Hore, D.K. Russell, Radical pathways in the thermal decomposition of pyridine and diazines: a laser pyrolysis and semi-empirical study, J. Chem. Soc.-Perkin Trans. 2 (1998) 269-275.

[68] K.M. Pamidimukkala, R.D. Kern, M.R. Patel, H.C. Wei, J.H. Kiefer, HighTemperature pyrolysis of toluene, J. Phys. Chem. 91 (1987) 2148-2154.

[69] J.P. Boudou, J.I. Paredes, A. Cuesta, A. Martinez-Alonso, J.M.D. Tascon, Oxygen plasma modification of pitch-based isotropic carbon fibres, Carbon 41 (2003) 41-56. 
[70] J.R. Pels, F. Kapteijn, J.A. Moulijn, Q. Zhu, K.M. Thomas, Evolution of nitrogen functionalities in carbonaceous materials during pyrolysis, Carbon 33 (1995) 1641-1653.

[71] C. Hontoria-Lucas, A.J. Lopez-Peinado, J.D.D. Lopez-Gonzalez, M.L. RojasCervantes, R.M. Martin-Aranda, Study of oxygen-containing groups in a series of graphite oxides - physical and chemical characterization, Carbon 33 (1995) 1585-1592.

[72] S. Biniak, G. Szymanski, J. Siedlewski, A. Swiatkowski, The characterization of activated carbons with oxygen and nitrogen surface groups, Carbon 35 (1997) 1799-1810.

[73] P. Garcia, J.F. Espinal, C.S.M. de Lecea, F. Mondragon, Experimental characterization and molecular simulation of nitrogen complexes formed upon NO-char reaction at 270 degrees $\mathrm{C}$ in the presence of $\mathrm{H} 2 \mathrm{O}$ and O-2, Carbon 42 (2004) 1507-1515.

[74] J.L. Hueso, J.P. Espinos, A. Caballero, J. Cotrino, A.R. Gonzalez-Elipe, XPS investigation of the reaction of carbon with NO, O-2, N-2 and H2O plasmas, Carbon 45 (2007) 89-96.

[75] M.C. Ortega-Liebana, M.M. Encabo-Berzosa, A. Casanova, M.D. Pereboom, J.O. Alda, J.L. Hueso, J. Santamaria, Upconverting Carbon Nanodots from Ethylenediaminetetraacetic Acid (EDTA) as Near-Infrared Activated Phototheranostic Agents, Chem.-Eur. J. 25 (2019) 5539-5546.

[76] G.A.M. Hutton, B. Reuillard, B.C.M. Martindale, C.A. Caputo, C.W.J. Lockwood, J.N. Butt, E. Reisner, Carbon Dots as Versatile Photosensitizers for Solar-Driven Catalysis with Redox Enzymes, J. Am. Chem. Soc. 138 (2016) 16722-16730.

[77] B.C.M. Martindale, G.A.M. Hutton, C.A. Caputo, E. Reisner, Solar Hydrogen Production Using Carbon Quantum Dots and a Molecular Nickel Catalyst, J. Am. Chem. Soc. 137 (2015) 6018-6025. 
[78] M.C. Ortega-Liebana, J.L. Hueso, R. Arenal, J. Santamaria, Titania-coated gold nanorods with expanded photocatalytic response. Enzyme-like glucose oxidation under nearinfrared illumination, Nanoscale 9 (2017) 1787-1792.

[79] A. Cuesta, P. Dhamelincourt, J. Laureyns, A. Martinezalonso, J.M.D. Tascon, Raman microprobe studies on carbon materials, Carbon 32 (1994) 1523-1532.

[80] S. Maldonado, S. Morin, K.J. Stevenson, Structure, composition, and chemical reactivity of carbon nanotubes by selective nitrogen doping, Carbon 44 (2006) 1429-1437.

[81] A.P. del Pino, A.M. Villarroya, A. Chuquitarqui, C. Logofatu, D. Tonti, E. Gyorgy, Reactive laser synthesis of nitrogen-doped hybrid graphene-based electrodes for energy storage, J. Mater. Chem. A 6 (2018) 16074-16086.

[82] T. Han, T.X. Fan, S.K. Chow, D. Zhang, Biogenic N-P-codoped TiO2: Synthesis, characterization and photocatalytic properties, Bioresour. Technol. 101 (2010) 6829-6835.

[83] M.C. Ortega-Liebana, J.L. Hueso, R. Fernandez-Pacheco, S. Irusta, J. Santamaria, Luminescent mesoporous nanorods as photocatalytic enzyme-like peroxidase surrogates, Chem. Sci. 9 (2018) 7766-7778.

[84] Z.F. Zhu, S.D. Wang, Y.J. Chang, D.B. Yu, Y. Jiang, Direct photodissociation of toluene molecules to photoluminescent carbon dots under pulsed laser irradiation, Carbon 105 (2016) 416-423.

[85] Q. Liu, D.T. Li, Z.F. Zhu, S.M. Yu, Y. Zhang, D.B. Yu, Y. Jiang, N-doped carbon dots from phenol derivatives for excellent colour rendering WLEDs, RSC Adv. 8 (2018) 4850-4856. [86] A.C. Ferrari, D.M. Basko, Raman spectroscopy as a versatile tool for studying the properties of graphene, Nat. Nanotechnol. 8 (2013) 235-246.

[87] T.Q. Lin, I.W. Chen, F.X. Liu, C.Y. Yang, H. Bi, F.F. Xu, F.Q. Huang, Nitrogen-doped mesoporous carbon of extraordinary capacitance for electrochemical energy storage, Science $350(2015)$ 1508-1513. 
[88] P. Wu, W. Li, Q. Wu, Y.S. Liu, S.X. Liu, Hydrothermal synthesis of nitrogen-doped carbon quantum dots from microcrystalline cellulose for the detection of $\mathrm{Fe} 3+$ ions in an acidic environment, RSC Adv. 7 (2017) 44144-44153.

[89] H.Q. Tao, K. Yang, Z. Ma, J.M. Wan, Y.J. Zhang, Z.H. Kang, Z. Liu, In Vivo NIR Fluorescence Imaging, Biodistribution, and Toxicology of Photoluminescent Carbon Dots Produced from Carbon Nanotubes and Graphite, Small 8 (2012) 281-290.

[90] T.H. Yang, L.D. Huang, Y.W. Harn, C.C. Lin, J.K. Chang, C.I. Wu, J.M. Wu, High Density Unaggregated $\mathrm{Au}$ Nanoparticles on ZnO Nanorod Arrays Function as Efficient and Recyclable Photocatalysts for Environmental Purification, Small 9 (2013) 3169-3182.

[91] V. Nguyen, J.H. Si, L.H. Yan, X. Hou, Electron-hole recombination dynamics in carbon nanodots, Carbon 95 (2015) 659-663.

[92] V. Nguyen, J.H. Si, L.H. Yan, X. Hou, Direct demonstration of photoluminescence originated from surface functional groups in carbon nanodots, Carbon 108 (2016) 268-273.

[93] V. Nguyen, L.H. Yan, H.H. Xu, M.M. Yue, One-step synthesis of multi-emission carbon nanodots for ratiometric temperature sensing, Appl. Surf. Sci. 427 (2018) 1118-1123.

[94] S.J. Zhu, Y.B. Song, J. Wang, H. Wan, Y. Zhang, Y. Ning, B. Yang, Photoluminescence mechanism in graphene quantum dots: Quantum confinement effect and surface/edge state, Nano Today 13 (2017) 10-14.

[95] Y.P. Sun, B. Zhou, Y. Lin, W. Wang, K.A.S. Fernando, P. Pathak, M.J. Meziani, B.A. Harruff, X. Wang, H.F. Wang, P.J.G. Luo, H. Yang, M.E. Kose, B.L. Chen, L.M. Veca, S.Y. Xie, Quantum-sized carbon dots for bright and colorful photoluminescence, J. Am. Chem. Soc. 128 (2006) 7756-7757.

[96] K.P. Gong, F. Du, Z.H. Xia, M. Durstock, L.M. Dai, Nitrogen-Doped Carbon Nanotube Arrays with High Electrocatalytic Activity for Oxygen Reduction, Science 323 (2009) 760-764. 
[97] S.Y. Wang, D.S. Yu, L.M. Dai, Polyelectrolyte Functionalized Carbon Nanotubes as Efficient Metal-free Electrocatalysts for Oxygen Reduction, J. Am. Chem. Soc. 133 (2011) $5182-5185$.

[98] L.W. Zhang, H.B. Fu, Y.F. Zhu, Efficient TiO2 photocatalysts from surface hybridization of TiO2 particles with graphite-like carbon, Adv. Funct. Mater. 18 (2008) 21802189.

[99] S. Banerjee, D.D. Dionysiou, S.C. Pillai, Self-cleaning applications of TiO2 by photoinduced hydrophilicity and photocatalysis, Appl. Catal. B-Environ. 176 (2015) 396-428.

[100] M.O. Valappil, V.K. Pillai, S. Alwarappan, Spotlighting graphene quantum dots and beyond: Synthesis, properties and sensing applications, Appl. Mater. Today 9 (2017) 350-371. [101] B.C.M. Martindale, E. Joliat, C. Bachmann, R. Alberto, E. Reisner, Clean Donor Oxidation Enhances the H-2 Evolution Activity of a Carbon Quantum Dot-Molecular Catalyst Photosystem, Angew. Chem.-Int. Edit. 55 (2016) 9402-9406.

[102] B. Bajorowicz, M.P. Kobylanski, A. Golabiewska, J. Nadolna, A. Zaleska-Medynska, A. Malankowska, Quantum dot-decorated semiconductor micro- and nanoparticles: A review of their synthesis, characterization and application in photocatalysis, Adv. Colloid Interface Sci. 256 (2018) 352-372.

[103] Y. Nosaka, A. Nosaka, Understanding Hydroxyl Radical ((OH)-O-center dot) Generation Processes in Photocatalysis, ACS Energy Lett. 1 (2016) 356-359. 